\title{
Artificial Neural Networks in Forensic Medicine (review)
}

\author{
KotsyubynskaYu.Z. ${ }^{1}$, Kozan N.M. ${ }^{1}$, Zelenchuk G.M. ${ }^{1}$, Voloshinovich V.M ${ }^{1}$, Kotsyubynsky A.O. ${ }^{2}$ \\ ${ }^{1}$ Ivano-Frankivsk National Medical University, Ivano-Frankivsk, Ukraine, ${ }^{2}$ Ivano-Frankivsk National Technical \\ University of Oil and Gas, Ivano-Frankivsk, Ukraine
}

\begin{abstract}
Thearticle deals with the possibilities and limitations of the use of artificial neural networks in forensic medical practice. It is known that in the work of forensic medicine experts have to process and evaluate a large amount of information, which may belong to different types of data - the site inspection protocols, photographic materials, macroscopic data obtained during the autopsy of the corpse, the results of laboratory tests, medical records etc. All the received data should be studied, categorized and evaluated according to international standards. Modern computer technologies of artificial intelligence (artificial neural networks) can help in the handling of forensic medicine data, which, in turn, will reduce to a minimum the probability of mistakes in preparation of expert conclusions. The algorithms used in artificial neural networks, as a result of processing different types of input data, can direct them to the resulting categorized outputs and structure them.
\end{abstract}

Keywords : artificial neural networks, artificial intelligence, forensic medicine, identification of a person.

\section{Introduction}

Recently, more and more researchers are using artificial intelligence elements in their practice. ${ }^{[1,2]}$ There are discussions whether the machine intelligence can replace doctors. [3] The scientific community converges in opinion, that in the nearest future this will not happen, but artificial intelligence could help doctors to make better decisions or even partially displace human judgment in certain narrow areas. The growing amount of data that can be used in medical practice, as well as the analytical methods used in their processing, allow us to give successful examples of the use of artificial intelligence in medicine. ${ }^{[4,5,6]}$ These algorithms are used in such areas like cardiology, dermatology, immunology, oncology, dentistry, genetics and so on. ${ }^{[1-8]}$ Elements of artificial intelligence begin to apply in

\section{Corresponding Author :}

Kotsyubynsky A.O.,

Ivano-Frankivsk National Technical University of Oil

and Gas, Ivano-Frankivsk, Ukraine

Contact : +380978762897

Email : kotsyubynskyao@gmail.com forensic medicine. ${ }^{[9-12]}$

The purpose of this work is to analyze the possibilities and limitations of the use of artificial neural networks in forensic medical practice (Fig. 1

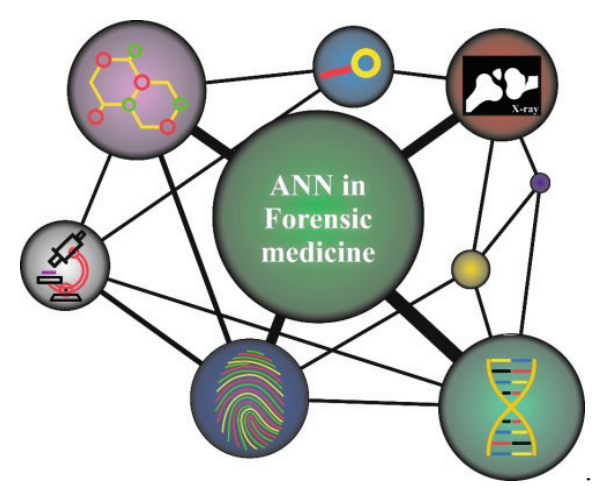

Fig. 1. The fields of possible application of ANN in forensic medicine.

Artificial neural networks.

Artificial neural network (ANN) -is a mathematical model, which in its structure is similar to the human nervous system. In the same way as the human being, ANN can learn and generalize knowledge. That is why ANN is refers to artificial intelligence. ANN is widely used in all fields of science and technology, and you 
even use it every day entering Facebookor looking for information in Google. There are many different types of ANN, which are concentrated on solving of the certain types of problems. ${ }^{[13]}$

In general, ANN is a system of connected and interacting processors (neurons). A neuron (the base element of $\mathrm{ANN}$ ) is a simple computing processor that can receive, process and transmit the information. By combining a large number of neurons into a single network, the system can solve non-trivial tasks.

Neurons in ANN are combined in layers (Fig. 2) the input layer (a set of neurons that receive information), n-hidden layers (the set of neurons, which will process the information), and the output layer (neurons which outputs the result).

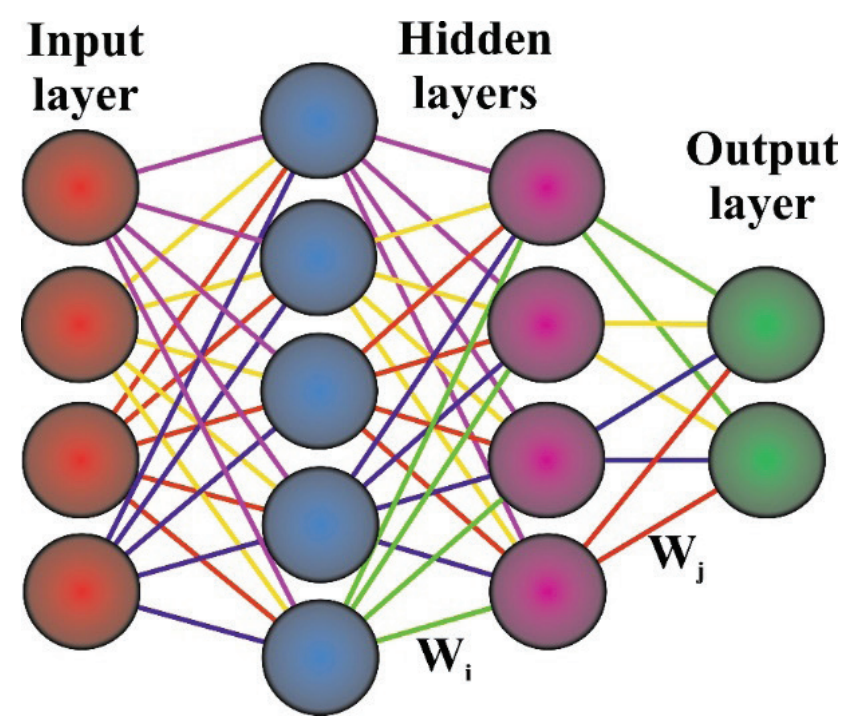

Fig. 2 .The Structureof the neuralnetwork.

During the operation, neurons operate with numbers. Usually these numbers are in the range $[0,1]$ or $[1,1]$. Each neuron has two parameters: inputdata and output data. The field of inputdata contains the summary information from all the neurons of the previous layer. After receiving the information, it is normalized by the activation function $\mathrm{f}(\mathrm{x})$, after which it falls into the field of output data. It should be noted, thatfor the input layer of neurons input information is equal to the output information(input $=$ output).

All theneurons are integrated into the network with the help of synapses (Fig. 2, $\mathrm{W}_{\mathrm{i}}, \mathrm{W}_{\mathrm{j}}$ ) . A synapse is a connection between two neurons that is characterized by the weightof the synapse. ${ }^{[14]}$ Due to the weight of the synapse, the input information changes during the transmission from one neuron to another. In Fig. 3 it is showed how the synapse weight can change the data in colors.During processing ofthe input data will be obtained the result in which the most important role will play the synapse with the most weight. The combination of all the weights of the synapse neurons allows the system to make the decisions. Depending on the complexity of the task, the number of neurons and layers can change a lt.

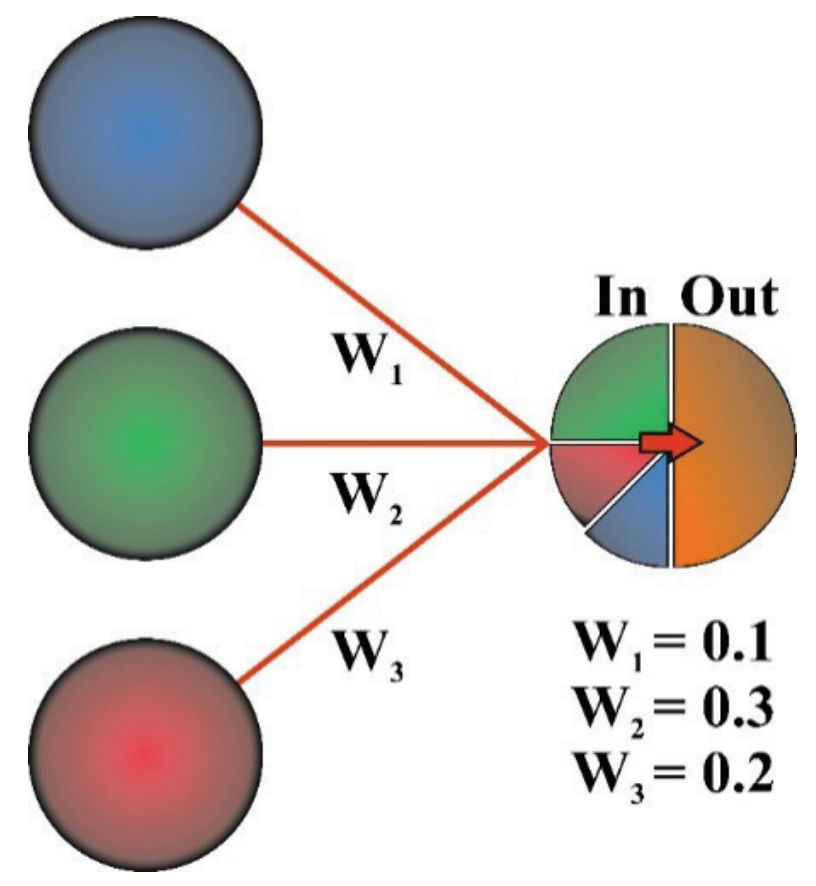

Fig. 3. Given the neural result of the processing of data by a neuron by the weight of synapses.

Another important element of the neural network is a function of activation (activation functions ${ }^{[15]}$ ) - the function which normalizes the input data (the function which allows you to interpret data in the form of numbers, belonging to the range $[0,1])$. In this activation function for determination of the output data, the total sum of input data and weight coefficients are compared with some threshold. If the sum is greater than the threshold, the processing element generates a signal, otherwise the signal is not generated (or a brake signal is generated). ${ }^{[16]}$ The most common practice is the using of the sigmoid function (Fig. 4). An important feature of sigmoid is the continuity of functions and their derivatis. 


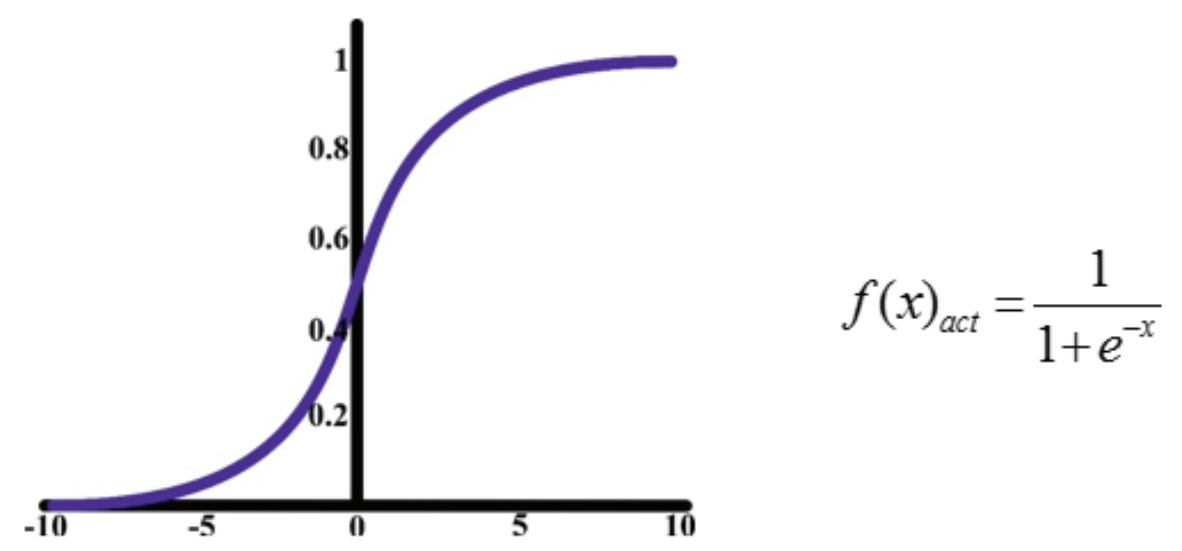

Fig. 4. Nonlinear activation function that approximates the minimum and maximum values in asymptotes.

In order the ANN could work, it must first be trained. Training is usually conducted on the databases with relevant input and output data. The data basis consists of already known cases and conclusions to them. Data can be both numeric and non-numeric. In the case of non-numerical data, they need to be structured by certain we categories.

Table1.The example of the input data table for teaching ANN. The order number corresponds to a certain person. Data $\mathrm{ji}_{\mathrm{i}}$ - data, fixed in a specific case and grouped into appropriate categories.

\begin{tabular}{|c|c|c|c|c|c|}
\hline $\mathbf{N}$ & \multicolumn{5}{|c|}{ Data } \\
\hline 1 & Data 11 & Data 12 & $\ldots$ & Data $1 \mathrm{i}$ & Result 1 \\
\hline 2 & Data 21 & Data 22 & $\ldots$ & Data $2 \mathrm{i}$ & Result 1 \\
\hline 3 & Data 31 & Data 32 & $\ldots$ & Data $3 \mathrm{i}$ & Result 2 \\
\hline$\ldots$ & $\ldots$ & $\ldots$ & $\ldots$ & $\ldots$ & $\ldots$ \\
\hline $\mathrm{j}$ & Data j1 & Data j2 & $\ldots$ & Data ji & Result 1 \\
\hline
\end{tabular}

The filling of the table can be illustrated by identifying racial affiliation of unknown male person. Table 2 .

Tab. 2: The example offilling the table with data to identify the race of unknown male person.

\begin{tabular}{|c|c|c|c|c|c|c|}
\hline $\mathbf{N}$ & Skin color & Eye color & HairColor & $\ldots$ & Growth & Result \\
\hline 1 & Yellow & Hazel & Black & $\ldots$ & 167 & Asian \\
\hline 2 & White & Blue & Blond & $\ldots$ & 178 & Europeoid \\
\hline 3 & Yellow & Hazel & Black & $\ldots$ & 175 & Asian \\
\hline 4 & White & Hazel & Black & $\ldots$ & 180 & Europeoid \\
\hline.. & $\ldots$ & $\ldots$ & $\ldots$ & $\ldots$ & $\ldots$ & Asian \\
\hline$j$ & Yellow & Hazel & Black & $\ldots$ & 174 & \\
\hline
\end{tabular}




\section{Softwarefor ANN building}

The power of modern computers allows to process rather difficult tasks even on the non-specialized devices. Experts can create and train ANN on conventional computers, using common software. There are many software packages available to work with ANN. [17] Most of them arefocused on the experienced user, who has programming skills.

Among the software with the low entering barrier can be noted the Statistica. ${ }^{[18]}$ This program allows you quickly build ANNs of different architecture and complexity. It is also important that after teaching ANN in this software, you can immediately conduct researches on new data and instantly get results.

\section{The practical use of $A N N$}

In its practice, forensic expert faces with many challenges of different complexity. Some of these tasks can be facilitated by the use of artificial intelligence.

\section{DNA studies}

Genetic identification is one of the main modern methods of analysis in forensic medicine. Thus, the researches of electropherograms are held in a huge quantityin forensic DNA laboratories.Genetic experts should be involved in the correct interpretation of the obtained data. ${ }^{[19]}$

In the work ${ }^{[20]}$, we can see data concerning the using ofANN for the identification and classification of peaks in the electropherogram. The authors note that the process of interpretation of the electropherogram can be time consuming and depends on the subjective evaluation of the analyst. These works show the possibility of using artificial neural networks for reading complex and mixed electrophoretic data. Thus, the use of ANN can simplify processing and improve the accuracy of DNA testing.

Authors ${ }^{[21]}$ showed the possibility to determine the age of the donor with the restored biological material, using the methods of DNA analysis and ANN. This information can be of considerable value for forensic investigations. The authors claim that aging is a complex process, associated with different molecular modifications in cells that re accumulated throughout the life, caused by genetic and epigenetic factors. In order to generate the accurate model for predicting chronological age, the authors used age-specific DNA methylation models from the data of whole blood.Because of data processing using ANN, authors achieved a good prediction result with a 4-year error on the blind test.

\section{Firearms}

Another possible application of ANN in forensic medicine is to establish the similarity of the balls, fired from the firearms. Thus, in the work ${ }^{[22]}$ it is showed, how ANN can be used in the study of bullet tracks. Theabovementioned work ANN studied on the well-known data bases, and then made the processing of unknowns balls. As a result, the network showed very good results of comparison on the test sample.

\section{Causes of death}

Often in practice, we have cases when it is quite difficult to establish direct relationships between diseases and death, especially in the partial or complete absence of medical records of a person. The authors ${ }^{[23]}$ use an ANN for classifying cause of death from verbal autopsy. The efficiency of ANN models was compared with two other classification methods (physician examination and logistic regression) that were tested on the same sample with the same verbal autopsy data. As a result, the comparisons of ANNmodels were as accurate as the other methods used.

\section{Pharmaceutical fingerprinting}

ANN can be also successfully applied in laboratory analyzes of chemical substances. Work ${ }^{[24]}$ shows the possibility of ANN application in forensic toxicology. Thus, authorsmade chromatogram medicines investigations in biological fluids and compared them with the reference samples. The result shows that the received ANN can provide fast, accurateand consecutivetechnique,applied for searching of the chemical agents in biological fluids.

In the work of Zhu H.,the potential possibilities of ANN application for drugs investigation are showed. It is noted that artificial intelligence is a promising method to greatly reduce the cost and time of drug discovery 
by providing evaluations of drug molecules in the early stages of development. [25]

\section{Investigation of bones and teeth}

ANNs can be successfully used in identifying an unknown person on cystic fragments. Thus, in the work ${ }^{[26]}$ the authors use ANN during the determination of the gender and age of the unknown person according to the odontological parameters of the jaws. They point out that the use of ANN is promisingbecause automates and simplifies the method of sex and age installation with minimal errors. In the works ${ }^{[27,28]}$ the possibility of using ANN in the teethand bones research is explored.

\section{Fingerprints}

The research of the fingerprints is one of the basicidentification method in forensic medicine.In the works ${ }^{[29-30]}$ the possibility of classifying fingerprints with the help of ANN is investigated. Built ANN allowed to reduce the time of image processing. The authors [31] proposed the method of comparing fingerprints, including partial (incomplete) images. In the works ${ }^{[32,33]}$ authors use fingerprints for predicting sexual identity. The ANNs made it possible to classify gender with a probability of $97 \%$.

Of course, the practical application of ANN is not limited to these examples. Currently, there are prerequisites for the application of ANN in practically every field of forensic medicine. The main problem, which may face a forensic expert in choosing this method of research is the content of database for network training. It is also required that expert should understand fundamental statistical and mathematical methods.

\section{Conclusions}

As noted earlier, ANN can be applied practically in any section of forensic medicine. Their advantages are the ability to process large amounts of data, less likely missing of critically important data, and reducing decision time. Yet, at this stage we cannot do without human control. ANN is necessary, first of all, as a helper or tool, which is designed to help in making difficult decisions. But in any case, it does not diminish the potential benefit, which can provide the skillful use of elements of artificial intelligence.

\section{Research Funding: None}

\section{Financial support and sponsorship: Nil}

\section{Conflict of Interest: None}

Ethical Clearance:It was obtained from Ethics Committee of the Ivano-Frankivsk National Medical University before starting the study.

\section{References}

1. Esteva A, Kuprel B, Novoa R, Ko J, Swetter S, Blau $\mathrm{H}$ et al. Dermatologist-level classification of skin cancer with deep neural networks. Nature. 2017;542(7639):115-118. DOI: 10.1038/ nature 21056

2. Gulshan V, Peng L, Coram M, Stumpe M, Wu D, Narayanaswamy A et al. Development and Validation of a Deep Learning Algorithm for Detection of Diabetic Retinopathy in Retinal Fundus Photographs. JAMA. 2016;316(22):2402. DOI: 10.1001/jama.2016.17216

3. Darcy A, Louie A, Roberts L. Machine Learning and the Profession of Medicine. JAMA. 2016;315(6):551. DOI: 10.1001/jama.2015.18421

4. Dheeba J, Albert Singh N, Tamil Selvi S. Computer-aided detection of breast cancer on mammograms: A swarm intelligence optimized wavelet neural network approach. Journal of Biomedical Informatics. 2014;49:45-52. DOI: 10.1016/j.jbi.2014.01.010

5. Murdoch T, Detsky A. The Inevitable Application of Big Data to Health Care. JAMA. 2013;309(13):1351. DOI:10.1001/jama.2013.393

6. Schwendicke F, Golla $\mathrm{T}$, Dreher $\mathrm{M}$, Krois J. Convolutional neural networks for dental image diagnostics: A scoping review. Journal of Dentistry. 2019;91:103226. DOI: 10.1016/j. jdent.2019.103226

7. Amato F, López A, Peña-Méndez E, Vaňhara P, Hampl A, Havel J. Artificial neural networks in medical diagnosis. Journal of Applied Biomedicine. 2013;11(2):47-58. DOI: 10.2478/v10136-0120031-x 
8. Dorado-Díaz P, Sampedro-Gómez J, VicentePalacios V, Sánchez P. Applications of Artificial Intelligence in Cardiology. The Future is Already Here. Revista Española de Cardiología (English Edition). 2019;72(12):1065-1075.DOI: 10.1016/j. rec.2019.05.014

9. Bewes J, Low A, Morphett A, Pate F, Henneberg M. Artificial intelligence for sex determination of skeletal remains: Application of a deep learning artificial neural network to human skulls. Journal of Forensic and Legal Medicine. 2019;62:40-43. DOI: 10.1016/j.jflm.2019.01.004

10. Lefèvre $\mathrm{T}$. Big data in forensic science and medicine. Journal of Forensic and Legal Medicine. 2018;57:1-6. DOI: 10.1016/j.jflm.2017.08.001

11. Patil V, Vineetha R, Vatsa S, Shetty D, Raju A, Naik $\mathrm{N}$ et al. Artificial neural network for gender determination using mandibular morphometric parameters: A comparative retrospective study. Cogent Engineering. 2020;7(1). DOI: 10.1080/23311916.2020.1723783

12. Margagliotti G, Bollé T. Machine learning \& forensic science. Forensic Science International. 2019;298:138-139. DOI: $\quad 10.1016 / j$. forsciint.2019.02.045

13. Rojas R. Neural networks. Springer Science \& Business Media; 2013.ISBN: 978-3-642-61068-4

14. Haykin S. Neural networks and learning machines. New York: Prentice Hall; 2009. ISBN: 978-0-13147139-9

15. Specht D. Probabilistic neural networks. Neural Networks. 1990;3(1):109-118. DOI: 10.1016/08936080(90)90049-Q

16. Karlik B, Olgac AV. Performance analysis of various activation functions in generalized MLP architectures of neural networks. International Journal of Artificial Intelligence and Expert Systems. 2011;1(4):111-122. http://www.cscjournals.org/ manuscript/Journals/IJAE/Volume1/Issue4/IJAE26.pdf

17. Dagli C. Artificial Neural Networks for Intelligent Manufacturing. Dordrecht: Springer Netherlands; 1994. ISBN 978-94-011-0713-6

18. Neural Networks in Statistica Program [Internet]. TIBCO Software Inc. 2020 [cited 17 May 2020].
Available from: http://www.statsoft.com/textbook/ neural-networks/

19. Taylor D, Harrison A, Powers D. An artificial neural network system to identify alleles in reference electropherograms. Forensic Science International: Genetics. 2017;30:114-126. DOI: 10.1016/j. fsigen.2017.07.002

20. Taylor D, Powers D. Teaching artificial intelligence to read electropherograms. Forensic Science International: Genetics. 2016;25:10-18. DOI: 10.1016/j.fsigen.2016.07.013

21. Vidaki A, Ballard D, Aliferi A, Miller T, Barron L, Syndercombe Court D. DNA methylation-based forensic age prediction using artificial neural networks and next generation sequencing. Forensic Science International: Genetics. 2017;28:225-236. DOI: 10.1016/j.fsigen.2017.02.009

22. Banno A. Estimation of Bullet Striation Similarity Using Neural Networks. Journal of Forensic Sciences. 2004;49(3):1-5. DOI: 10.1520/ JFS2002361

23. Boulle A, Chandramohan D, Weller P. A case study of using artificial neural networks for classifying cause of death from verbal autopsy. International Journal of Epidemiology. 2001;30(3):515-520. DOI: $10.1093 /$ ije/30.3.515

24. Tetko I, Villa A, Aksenova T, Zielinski W, Brower J, Collantes E et al. Application of a Pruning Algorithm To Optimize Artificial Neural Networks for Pharmaceutical Fingerprinting. Journal of Chemical Information and Computer Sciences. 1998;38(4):660-668. DOI: 10.1021/ci970439j

25. Zhu H. Big Data and Artificial Intelligence Modeling for Drug Discovery. Annual Review of Pharmacology and Toxicology. 2020;60(1):573-589. DOI: 10.1146/annurevpharmtox-010919-023324

26. Patil V, Vineetha R, Vatsa S, Shetty DK, Raju A, Naik $\mathrm{N}$ et al. Artificial neural network for gender determination using mandibular morphometric parameters: A comparative retrospective study. Cogent Engineering. 2020;7(1). 1723783. DOI: $10.1080 / 23311916.2020 .1723783$

27. Park W, Park J. History and application of artificial neural networks in dentistry. European Journal of 
Dentistry. 2018;12(04):594-601. DOI: 10.4103/ ejd.ejd_325_18

28. du Jardin P, Ponsaillé J, Alunni-Perret V, Quatrehomme G. A comparison between neural network and other metric methods to determine sex from the upper femur in a modern French population. Forensic Science International. 2009;192(1-3):127. e1-127.e6. DOI: 10.1016/j.forsciint.2009.07.014

29. Nagaty K. Fingerprints classification using artificial neural networks: a combined structural and statistical approach. Neural Networks. 2001;14(9):1293-1305. DOI: 10.1016/s08936080(01)00086-7

30. Basturk A, Basturk N, Qurbanov O. Fingerprint recognition by deep neural networks and fingercodes. 26th Signal Processing and Communications Applications Conference (SIU).
IEEE; 2018. DOI: 10.1109/SIU.2018.8404577

31. Arada, G. P., \&Dadios, E. P. Partial fingerprint identification through checkerboard sampling method using ANN. TENCON 2012 IEEE Region 10 Conference. IEEE.2012. DOI: 10.1109/ TENCON.2012.6412170

32. Abdullah S, Rahman A, Abas Z, Saad W. Multilayer Perceptron Neural Network in Classifying Gender using Fingerprint Global Level Features. Indian Journal of Science and Technology. 2016;9(9). DOI: $10.17485 / \mathrm{ijst} / 2016 / \mathrm{v} 9 \mathrm{i} 9 / 84889$

33. Abdullah, S. F., Rahman, A. F. N. A., \& Abas, Z. A. Classification Of Gender By Using Fingerprint Ridge Density In Northern Part Of Malaysia. ARPN J. Eng. Appl. Sci. 2015; 10(22): 10722-10726. http://www.arpnjournals.org/jeas/research_papers/ rp_2015/jeas_1215_3122.pdf 